\title{
Compliance Control and Selective Compliance Center via Internal Forces in Redundantly Actuated Closed Chain Mechanisms
}

\author{
Mehmet Arif AdLI* and Hideo HANAFUSA*
}

\begin{abstract}
In closed chain mechanisms, internal forces can be generated and controlled by adding redundant active joints on the links. The internal forces do no work but have effect on the total stiffness of the mechanisms. The stiffness of a closed chain mechanism can be changed by internal forces without changing its inherent stiffness. The effect of the internal forces depends on the magnitude and mode of the internal forces as well as the change in the configuration of the mechanism. In this study, we explain how to achieve the compliance control in certain directions of motion and change the compliance center by properly generating internal forces for the selected configurations of a planar closed chain mechanism with one redundant actuation.
\end{abstract}

Key Words: closed chain mechanisms, internal forces, compliance, compliance center

\section{Introduction}

The terms "compliance" and "compliance center" are mostly used in assembly like applications where at least two parts have to be mated. In such cases it is important to locate the compliance center at a desired point and provide the appropriate compliance such that the objective task can be accomplished.

One way to locate the compliance center at a desired place and to provide the required compliance is to control the joint compliances simultaneously as suggested in Refs. 1) and 2). However, the required compliance can not always be achieved adequately due to the time delay in digital controllers and due to the limit on the compliance of the joints for the stability problems. Therefore, in the cases where the manipulators are required to perform fine motions, structural compliance devices like compliance wrist or RCC (Remote Center Compliance) are used. The RCC devices put the compliance center at the known location and provide the required compliance. The disadvantage of this devices is that, they are not programmable. They are suited for only the tasks that they are designed for. Parts with different geome-

\footnotetext{
* Faculty of Science and Engineering, Ritsumeikan University, 56-1 Kitamachi, Tojiin, Kita-ku, Kyoto (Received March 6, 1992)

(Revised August 17, 1992)
}

tries and different rigidities require different $\mathrm{RCC}$ devices. Apart from these physical compliance devices, in this paper we show that, internal forces can also be used to provide the required compliance and locate the compliance center at a desired location without changing the compliance of drive systems.

Although the internal forces that balance within the mechanisms do not contribute to the resultant force, they affect the stiffness of the mechanisms. The principle of the internal force effect on the stiffness of the closed mechanisms is extensively analyzed in Ref. 3 ). The analysis for redundantly actuated parallel manipulators can be seen in Ref. 4). Stiffness generation in overconstrained mechanisms has also been studied in Refs. 5) and 6). However, these works suggest but do not deal with the utilization of the internal forces in practical applications clearly.

There are also several studies in the field of grasping by multi-fingered hands in which the internal forces affect the stiffness of the grasping ${ }^{7) ~ 10)}$. The internal forces here are the grasping forces exerted by the fingers on the object. In these studies, the common conclusion is that, increasing the grasping forces may turn the grasp from a stable one into an unstable one due to the negative stiffness generated by these forces. In such cases, it has been suggested to increase the stiffness of the joints and/or fingertips to secure the stability. 
As indicated in Ref. 6), by adding sufficiently enough number of redundant actuators, the compliance of a closed chain mechanism can be actively controlled at any instant during the motion and the compliance center can be located anywhere on its end effector. However, to manage this requires six redundant actuators in addition to three actuators to drive the mechanism in $2 \mathrm{D}$ plane. This number increases to twenty one redundant actuators for 3D space. This is practically not feasible as it causes excess energy consumption and requires long controlling time as well as the placement of so many actuators. Therefore, for practical purposes it is necessary to reduce the required number of redundant actuators to reasonable numbers. This is essentially a design problem. It can be achieved by carefully considering the geometry of the mechanism.

In our previous study ${ }^{3}$, we defined the internal forces in closed chain mechanisms as "the force components that balance within a mechanism and do not produce any resultant force while the mechanism is at the equilibrium state and there is no external force acting". Based on this assumption we consider the internal forces that are generated by redundant actuation only.

Here in this study we used the internal forces in a planar closed chain mechanism to achieve the compliant motion and to locate the compliance center near the desired point while keeping the compliance of the drive systems unchanged. We utilized suitable geometrical configurations that reduce the number of non-zero compliance elements and eliminate the dependency between these elements. By this way we can control the elements of the compliance matrix which are related to the rotational motions via internal forces generated by only using one redundant actuator. Then we showed the existence of a selective range for the compliance center by generating appropriate internal forces.

\section{Compliance of a Closed Chain Mechanism}

The compliance of a mechanism, whether formed of an open or a closed chain, is generally due to the stiffness of the drive systems, transmission elements, links and in the case of contact, the stiffness of the environment as well. For the closed chain mecha- nisms, the internal forces are another factor that determine the overall compliance. Without considering the effect of the internal forces, the compliance due to the drive systems, that is, the compliance due to the active joints dominates the other factors. Therefore, usually the compliance of a robotic mechanism is attributed to the compliance of the drive systems only. Here, we will consider the compliance which is due to the drive systems and internal forces. To distinguish one from the other, we will call the compliance due to the drive systems as the "inherent compliance”. Although, in general the drive systems that generate the compliance of the mechanisms are controlled in joint space, the majority of the tasks performed by the robotic mechanisms take place in the work space coordinates. Therefore, it is necessary to represent the compliance of a mechanism in its work space, i.e., in the end effector coordinates. Throughout this study, for convenience in mathematical procedure, instead of compliance we will use the term "stiffness" (the inverse of compliance). Here, we will shortly explain the principle of stiffness generation due to the internal forces based on our previous papers 3) and 4).

Fig. 1 shows a planar closed chain mechanism which is composed of two arms and a floating link. Each arm has two degrees of freedóm and is driven by two actuators represented by two springs. The arms are connected to the floating link by passive joints. We assume that the arms driving the mechanism are initially free of constraints. The subscript $u$ is used to indicate the unconstrained condition. The internal force $\boldsymbol{F}_{I}=\left[\begin{array}{ll}\boldsymbol{f}_{I 1}^{T} & \boldsymbol{f}_{I 2}^{T}\end{array}\right]^{T}$ is generated when the arms are combined via floating link to form a closed chain. That is,

$$
\begin{aligned}
& \boldsymbol{f}_{I i}=\left(\boldsymbol{J}_{f i}^{-1}\right)^{T} \boldsymbol{k}_{q i}\left(\boldsymbol{q}_{e i}-\boldsymbol{q}_{u i}\right) \quad i=1,2 \\
& \boldsymbol{f}_{I 1}+\boldsymbol{f}_{I 2}=\mathbf{0}
\end{aligned}
$$

where,

$$
\begin{aligned}
& \boldsymbol{k}_{q_{i}}=\operatorname{diag}\left[k_{q_{i 1}} k_{q_{i 2}}\right]^{T} \in R^{2 \times 2}: \text { joint stiffness matrix } \\
& \text { of the } i^{\text {th }} \text { arm } \\
& \boldsymbol{J}_{f i}=\frac{\partial \boldsymbol{r}_{c i}}{\partial \boldsymbol{q}_{i}} \in R^{2 \times 2}: \text { Jacobian of the } i^{\text {th }} \text { arm } \\
& \boldsymbol{q}_{e i}=\left[\begin{array}{ll}
q_{e i 1} & q_{e i 2}
\end{array}\right]^{T} \in R^{2} \text { : active joint angles of the } i^{t h} \\
& \text { arm at closed form during } \\
& \text { static equilibrium }
\end{aligned}
$$




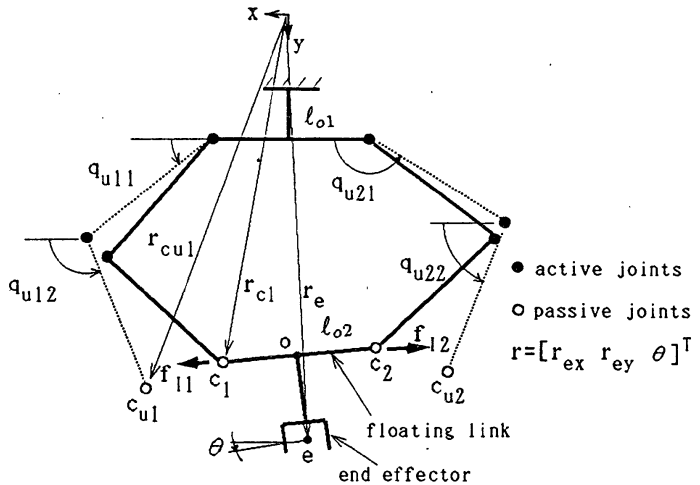

( a )

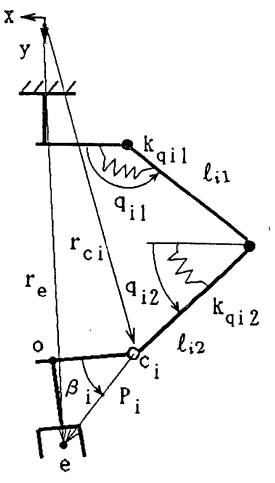

(b)

Fig. 1 A planar closed mechanism

$\boldsymbol{q}_{u i}=\left[\begin{array}{ll}q_{u i 1} & q_{u i 2}\end{array}\right]^{T} \in R^{2}$ : joint angles of the $i^{\text {th }}$ arm at free condition

The potential energy of the closed chain mechanism. due to the joint stiffnesses at an arbitrary state can be expressed by

$$
p_{E}=\frac{1}{2} \sum_{i=1}^{2}\left(\boldsymbol{q}_{i}-\boldsymbol{q}_{u i}\right)^{T} \boldsymbol{k}_{q_{i}}\left(\boldsymbol{q}_{i}-\boldsymbol{q}_{u i}\right)^{\prime}
$$

The stiffness of the mechanism in work space coordinates can be determined from,

$$
\boldsymbol{K}_{e}=\frac{\partial^{2} p_{E}}{\partial \boldsymbol{r}^{2}}
$$

where $\boldsymbol{r}=\left[\boldsymbol{r}_{e}^{T} \theta\right]^{T} \in R^{3}$ : position and orientation of the end effector

$r_{e}=\left[\begin{array}{ll}r_{e x} & r_{e y}\end{array}\right]^{T} \in R^{2}$ : position of the end effector

Note that, $r=f\left(\boldsymbol{q}_{1}, \boldsymbol{q}_{2}\right)$

Expanding ( 4 ) yields

$$
\begin{aligned}
\boldsymbol{K}_{e}= & \sum_{i=1}^{2} \boldsymbol{J}_{i}^{T} \boldsymbol{k}_{q i} \boldsymbol{J}_{i}+\sum_{i=1}^{2} \boldsymbol{J}_{o i}^{T}\left[\frac{\partial}{\partial \boldsymbol{q}_{i}}\left(\boldsymbol{J}_{f i}^{-1}\right)^{T}\right] \boldsymbol{k}_{q i}\left(\boldsymbol{q}_{i}-\boldsymbol{q}_{u i}\right) \boldsymbol{J}_{i} \\
& -\sum_{i=1}^{2}\left[\frac{\partial}{\partial \boldsymbol{r}} \boldsymbol{J}_{o i}^{T}\right]\left(\boldsymbol{J}_{f i}^{-1}\right)^{T} \boldsymbol{k}_{q i}\left(\boldsymbol{q}_{i}-\boldsymbol{q}_{u i}\right)
\end{aligned}
$$

where

$$
\begin{aligned}
\boldsymbol{J}_{o i}=\frac{\partial\left(\boldsymbol{r}_{e}-\boldsymbol{P}_{i}\right)}{\partial \boldsymbol{r}} \in R^{2 \times 3}: & \text { Jacobian relating the } i^{\text {th }} \\
& \text { arm tip to the end } \\
& \text { effector }
\end{aligned}
$$

$\boldsymbol{J}_{i}=\boldsymbol{J}_{f i}^{-1} \boldsymbol{J}_{o i} \in R^{2 \times 3}:$ Jacobian of the closed chain mechanism through joints of the $i^{\text {th }}$ arm

From eq. ( 1 ),

$$
\boldsymbol{q}_{u i}=\boldsymbol{q}_{e i}-\boldsymbol{k}_{q i}^{-1}\left(\boldsymbol{J}_{f i}^{T} \boldsymbol{f}_{I i}\right)
$$

Substituting eq. ( 6 ) into ( 5 ) yields the stiffness of the mechanism at equilibrium state

$$
\begin{aligned}
\boldsymbol{K}_{e}= & \sum_{i=1}^{2} \boldsymbol{J}_{i}^{T} \boldsymbol{k}_{q i} \boldsymbol{J}_{i}+\sum_{i=1}^{2} \boldsymbol{J}_{o i}^{T}\left[\frac{\partial}{\partial \boldsymbol{q}_{i}}\left(\boldsymbol{J}_{f i}^{-1}\right)^{T}\right]\left(\boldsymbol{J}_{f i}^{T} \boldsymbol{f}_{I i}\right) \boldsymbol{J}_{i} \\
& -\sum_{i=1}^{2}\left[\frac{\partial}{\partial \boldsymbol{r}} \boldsymbol{J}_{o i}^{T}\right] \boldsymbol{f}_{I i}
\end{aligned}
$$

here,

$$
\boldsymbol{k}_{e}=\sum_{i=1}^{2} \boldsymbol{J}_{i}^{T} \boldsymbol{k}_{q i} \boldsymbol{J}_{i}
$$

is the inherent stiffness of the mechanism defined at the end effector. Note that, we neglect the stiffness that may arise due to the flexibility of the structure of the mechanism, and assume that the inherent stiffness is the stiffness provided at joints.

$$
\Delta \boldsymbol{k}_{e}^{*}=\sum_{i=1}^{2} \boldsymbol{J}_{o i}^{T}\left[\frac{\partial}{\partial \boldsymbol{q}_{i}}\left(\boldsymbol{J}_{f i}^{-1}\right)^{T}\right]\left(\boldsymbol{J}_{f i}^{T} \boldsymbol{f}_{l i}\right) \boldsymbol{J}_{i}
$$

is the additional stiffness due to the effect of internal forces on the stiffness of each arm (see Fig. 2(a)). Equation (9) can also be written as

$$
\Delta \boldsymbol{k}_{e}^{*}=\sum_{i=1}^{2} \boldsymbol{J}_{i}^{T}\left[\frac{\partial}{\partial \boldsymbol{q}_{i}}\left(\boldsymbol{J}_{f i}\right)^{T}\right] \boldsymbol{f}_{I i} \boldsymbol{J}_{i}
$$

where $\left[\frac{\partial}{\partial \boldsymbol{q}_{i}}\left(\boldsymbol{J}_{f i}\right)^{T}\right] \boldsymbol{f}_{I i}$ is the additional joint stiffness on the $i^{\text {th }}$ arm due to the internal force $\boldsymbol{f}_{I i}$.

$$
\Delta \boldsymbol{k}_{e}^{* *}=-\sum_{i=1}^{2}\left[\frac{\partial}{\partial \boldsymbol{r}} \boldsymbol{J}_{o i}^{T}\right] \boldsymbol{f}_{I i}
$$

is the direct effect of the internal forces on the stiffness of the floating link (see Fig. 2 (b)).

The stiffness terms in eqs. (10) and (11) are called as "the effect of changes in grasp geometry" in Ref. 9).

Although the equations above are written for a planar closed chain mechanism, by small changes they can also apply to general spatial closed chain mechanisms. 


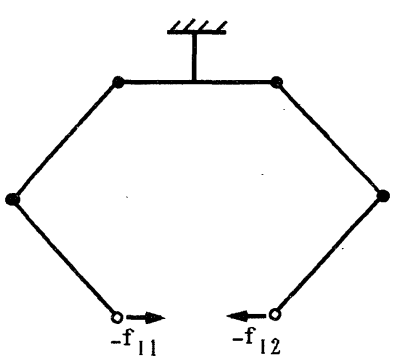

(a)

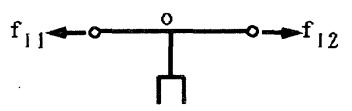

(b)

Fig. 2 Internal forces acting on the arms and the floating link

The additional stiffnesses $\Delta \boldsymbol{k}_{e}^{*}$ and $\Delta \boldsymbol{k}_{e}^{* *}$ depend on the magnitude and mode (i.e., compressive or tensile) of the internal forces and the change in the configuration of the mechanism. However, since the arms are passively connected to the floating link, the relative position between the end effector and each arm tip remains unchanged. This eliminates the dependency of $\Delta \boldsymbol{k}_{e}^{* *}$ to the change in the configuration of the mechanism. Hence, $\Delta \boldsymbol{k}_{e}^{* *}$ is the function of internal forces only and includes only the element for rotational motion. That is,

$$
\Delta \boldsymbol{k}_{e}^{* *}=\left[\begin{array}{ccc}
0 & 0 & 0 \\
0 & 0 & 0 \\
0 & 0 & \Delta k_{\theta}^{* *}
\end{array}\right]
$$

In the cases when the floating link is not passively connected to the arm tips, (as in the case of grasping by multi-fingered hands) and there is rolling, $\Delta \boldsymbol{k}_{e}^{* *}$ includes not only rotational elements but also elements for the other directions ${ }^{9)}$.

$\Delta k_{\theta}^{* *}$ is positive if the internal forces acting on the floating link are tensile and negative if the internal forces are compressive. Unlike $\Delta \boldsymbol{k}_{e}^{* *}, \Delta \boldsymbol{k}_{e}^{*}$ is strictly dependent on the configuration of the mechanism. In general, it includes the elements for all directions, that is,

$$
\Delta \boldsymbol{k}_{e}^{*}=\left[\begin{array}{ccc}
\Delta k_{x}^{*} & \Delta k_{x y}^{*} & \Delta k_{x \theta}^{*} \\
\Delta k_{y x}^{*} & \Delta k_{y}^{*} & \Delta k_{y \theta}^{*} \\
\Delta k_{\theta x}^{*} & \Delta k_{\theta y}^{*} & \Delta k_{\theta}^{*}
\end{array}\right] \begin{gathered}
\Delta k_{x y}^{*}=\Delta k_{y x}^{*} \\
\Delta k_{\theta x}^{*}=\Delta k_{x \theta}^{*} \\
\Delta k_{y \theta}^{*}=\Delta k_{\theta y}^{*}
\end{gathered}
$$

All these elements are in general dependent on each other. To reduce the number of non-zero elements in

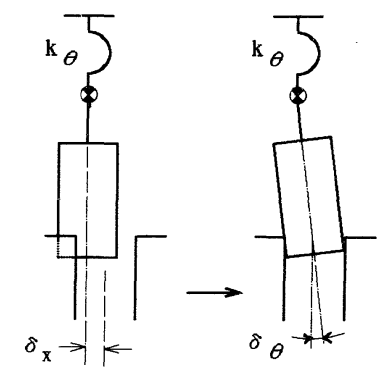

Fig. 3 Transformation of lateral errors into rotational errors

$\Delta \boldsymbol{k}_{e}^{*}$ we will utilize special geometrical configurations.

\section{Utilization of Internal Forces in Compli- ance Control}

The total stiffness in eq. ( 7 ) which was derived for the mechanism shown in Fig. 1 , is a $(3 \times 3)$ symmetric matrix with six variable elements. In order to control all these elements independently by means of internal forces while keeping the inherent stiffness unchanged, we need six redundant actuators to generate the required internal forces ${ }^{6}$. This is practically not feasible. In practical applications as in the case of assembly operations, we need not to control all the elements of the compliance matrix. In assembly operations, where at least two parts are in contact, the most critical motions are in the form of small rotational movements. In majority of the cases as in the case of peg-in-hole insertion, the translational errors are converted into the rotational errors (Fig. 3). This indicates that, the control of compliance elements for the rotational motions are primarily important. Especially in assembly applications, the successfulness of the task is almost totally dependent on the accuracy of the rotational movements. Therefore, the emphasis in this study is given to the utilization of the internal forces in the control of the compliance elements for the rotational motion only.

Assuming that the two arms have the same link lengths and same joint stiffnesses, we can combine the arms in different ways to form a closed chain mechanism as shown in Fig. 4. From eqs. ( 8 ) and (9), $\boldsymbol{k}_{e}$ and $\Delta \boldsymbol{k}_{e}^{*}$ for each of these configurations are as follows. Note that, $\boldsymbol{k}_{e}$ and $\Delta \boldsymbol{k}_{e}^{*}$ are defined for a point "e" at 
the end effector while $r_{e x}=0,\left(r_{e y}-r_{o y}\right)>0$ and $\theta_{e}=0$ with respect to the reference frame. For Fig. 4 (a)

$$
\left.\boldsymbol{k}_{e}=\left[\begin{array}{ccc}
\oplus & 0 & \oplus \\
0 & \oplus & 0 \\
\oplus & 0 & \oplus
\end{array}\right], \Delta \boldsymbol{k}_{e}^{*}= \pm\left[\begin{array}{ccc}
\ominus & 0 & \oplus \\
0 & \oplus & 0 \\
\oplus & 0 & \oplus
\end{array}\right]\right]
$$

For Fig. 4( b )

$$
\boldsymbol{k}_{e}=\left[\begin{array}{ccc}
\oplus & 0 & \oplus \\
0 & \oplus & 0 \\
\oplus & 0 & \oplus
\end{array}\right], \Delta \boldsymbol{k}_{e}^{*}= \pm\left[\begin{array}{ccc}
\oplus & 0 & \oplus \\
0 & \theta & 0 \\
\oplus & 0 & \ominus
\end{array}\right]
$$

For Fig. 4( c)

$$
\begin{gathered}
\boldsymbol{k}_{e}=\left[\begin{array}{ccc}
\oplus & 0 & \oplus \\
0 & \oplus & 0 \\
\oplus & 0 & \oplus
\end{array}\right], \Delta \boldsymbol{k}_{e}^{*}= \pm\left[\begin{array}{ccc}
0 & 0 & \oplus \\
0 & 0 & 0 \\
\oplus & 0 & \oplus
\end{array}\right] \\
\Delta \boldsymbol{k}_{e}^{* *}= \pm\left[\begin{array}{lll}
0 & 0 & 0 \\
0 & 0 & 0 \\
0 & 0 & \oplus
\end{array}\right] \text { for all three cases }
\end{gathered}
$$

Here,

+ : corresponds to tensile internal forces and

- : corresponds to compressive internal forces.

$\oplus$ and $\Theta$ indicates whether the element has positive or negative value.

These are the three basic configurations which give the minimum number of non-zero elements for $\boldsymbol{k}_{e}$ and $\Delta \boldsymbol{k}_{e}^{*}$. However it is further possible to obtain some more configurations which reduce the number of non-zero stiffness elements by changing the link lengths or the inherent joint stiffnesses. As understood from eq. (13), the configuration which leads to the least non-zero additional stiffness elements is that of Fig. 4(c). Furthermore, the additional stiffness elements in this case are all related to the rotational motion.

For the assembly applications, generally the range of the motion where the compliance control is required is relatively small compared to the whole motion range. In addition to that during the compliant phase of the motion the deviations in position are very small, therefore, the geometrical configuration of the manipulator is not changed too much. In short, the motion can be assumed as quasi-static. Based on this fact, we can use the stiffness values computed for the static equilibrium state.

From eqs. (9) and (11), the total change in rotational stiffness due to the internal forces is,

$$
\Delta K_{\theta}=\Delta k_{\theta}^{*}+\Delta k_{\theta}^{* *}
$$

Let $k_{\theta}$ be the inherent rotational stiffness of the mechanism at the end effector computed from eq. (8). If $\Delta K_{\theta}$ is generated such that, the total rotational stiffness of the mechanism, $K_{\theta}$ is,

$$
K_{\theta}=k_{\theta}+\Delta K_{\theta} \cong 0
$$

then, the end effector will attain a very high rotational compliance which is quite useful during assembly operations. Note however that, while maintaining eq. (15) an additional condition

$$
K_{\theta}>0
$$

must be satisfied in order to secure the stability of the motion. For the quasi-static motion, the dependency of $\Delta K_{\theta}$ to the change in configuration of the mechanism can be neglected. Hence, $\Delta K_{\theta}$ can be changed by only changing the internal forces. Since the inherent stiffness matrix $\boldsymbol{k}_{e}$ is a positive definite matrix, $k_{\theta}>0$ always. Therefore, to achieve the condition in eq.

(15) we must have

$$
\Delta k_{\theta}^{*}+\Delta k_{\theta}^{* *}<0
$$

$\Delta k_{\theta}^{* *}<0$ if the internal forces are compressive. However, the positiveness or negativeness of $\Delta k_{\theta}^{*}$ depends

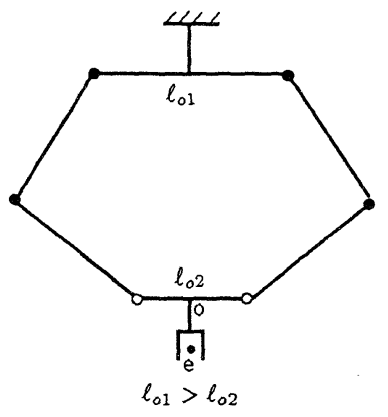

(a)

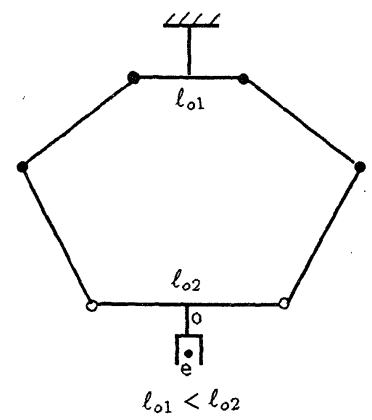

(b)

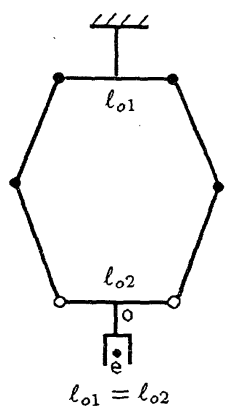

(c)

Fig. 4 Geometric configurations giving the least non-zero stiffness elements 
on the geometrical configuration of the mechanism as can be seen in eq. (13). If we select a geometry similar to that of Fig. $4(\mathrm{c})$, then, $\Delta k_{\theta}^{*}<0$ as well for the compressive internal forces. If the mechanism is operated at that configuration, to satisfy the condition in eq. (15), the magnitude of the internal force is obtained, by determining $k_{\theta}, \Delta k_{\theta}^{*}$ and $\Delta k_{\theta}^{* *}$ from eqs. ( 8 ), ( 9 ) and (11) and then substituting into eq. (15), as follows

$$
\begin{array}{r}
f_{I}=\frac{K_{\theta d}-\sum_{i=1}^{2} P^{2}\left[k_{s i x} \sin ^{2} \beta+k_{s i y} \cos ^{2} \beta\right]}{\left\{-\frac{1}{2} \sum_{i=1}^{2} P^{2}\left[\sin \left(2 q_{i 2}\right) \cos q_{i 1}\right.\right.} \\
\left.\left.+\sin \left(2 q_{i 1}\right) \cos q_{i 2}\right]\left(a_{i}\right)^{2} l^{3} \sin (2 \beta)\right\}+l_{o 2}
\end{array}
$$

where

\section{$K_{\theta d}:$ desired rotational stiffness}

$P=\left\|\boldsymbol{P}_{i}\right\|:$ distance between each arm tip and the end effector $\left(P_{1}=P_{2}=P\right)$

$\beta$ : (see Fig. 1) $\left(\beta_{1}=\beta_{2}=\beta\right)$

$l$ : length of the links on the arms

$l_{o 2}$ : length of the floating link

$a_{i}=-\frac{1}{l^{2} \sin \left(q_{i 1}-q_{i 2}\right)}, i=1,2$

$k_{\text {six }}, k_{\text {siy }}$ : diagonal elements of the stiffness matrix at the $i^{\text {th }}$ arm tip (see Ref. 4))

$$
\begin{aligned}
& k_{s i x}=\left(a_{i} l\right)^{2}\left[\cos ^{2}\left(q_{i 2}\right) k_{q i 1}+\cos ^{2}\left(q_{i 1}\right) k_{q i 2}\right] \\
& k_{s i y}=\left(a_{i} l\right)^{2}\left[\sin ^{2}\left(q_{i 2}\right) k_{q i 1}+\sin ^{2}\left(q_{i 1}\right) k_{q i 2}\right]
\end{aligned}
$$

The internal force $f_{I}$ must not violate eq. (16) and must be within the allowable range limited by the characteristics of the mechanism. Therefore, the geometrical configuration of the mechanism at which the compliant motion is going to be done must be selected such that, eq. (15) can be satisfied with the internal forces within this allowable range. Fig. 5 (a) shows the inherent rotational stiffness at the end effector, and the three different desired rotational stiffnesses in the range from $r_{e y}=0.24 m$ to $r_{e y}=0.39$ $m$ (see Fig. 5(b)). The required internal forces to realize these desired rotational stiffnesses are shown in Fig. 6 It is clear that to obtain the desired rotational stiffnesses at the extended configurations of the mechanism requires extremely large internal forces.

The values of joint stiffnesses and the link lengths for the Figs. 5 and 6 were selected as

$$
\left.\begin{array}{l}
k_{q i 1}=k_{q_{i 2}}=k_{q}=2.05 \mathrm{Nm} / \mathrm{rad} \\
l_{i 1}=l_{i 2}=l=0.128 \mathrm{~m} \\
l_{o i}=l_{o}=0.128 \mathrm{~m}
\end{array}\right\} \quad i=1,2
$$

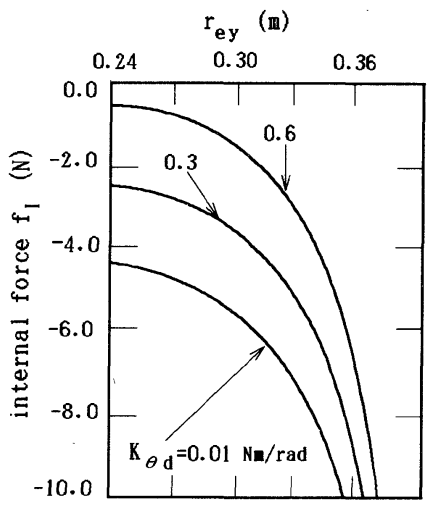

Fig. 6 Required internal force to realize the desired rotational stiffness

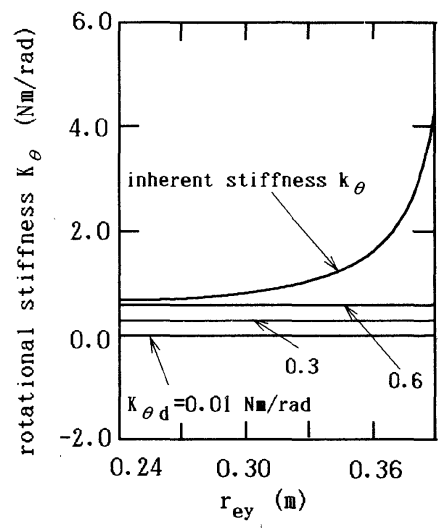

(a)

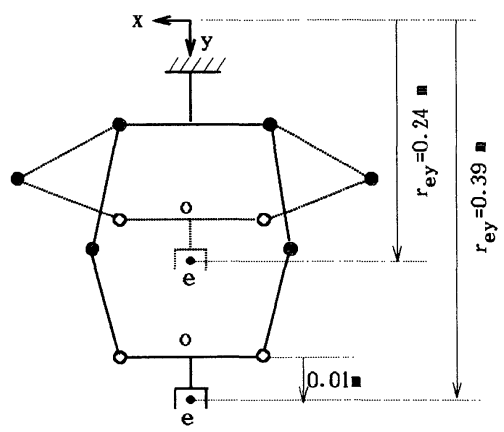

(b)

Fig. 5 Inherent and desired rotational stiffnesses 


\section{Selective Compliance Center via Internal Forces}

The compliance center of a mechanism is located at such a point that, any arbitrary force passing through this point yields only translational displacement along the direction of the applied force (Fig. 7). In the case of assembly applications where the mechanism is in contact with the environment, the compliance center must be as close to contact point as possible in order to achieve the assembly process smoothly. Without using the physical compliance devices like RCC or compliant wrist, the compliance center of a joint space controlled manipulator is dependent to the stiffness of the joints and the geometry and kinematics of the manipulator. Taking only the inherent joint stiffnesses into account, the compliance center of a manipulator is located at a point such that the stiffness,

$$
\boldsymbol{k}=\sum_{i=1}^{2} \boldsymbol{J}_{i}^{T} \boldsymbol{k}_{q i} \boldsymbol{J}_{i}
$$

at that point is diagonal. We call this point as the "inherent compliance center". In the case of no internal forces, the compliance center and the inherent compli-

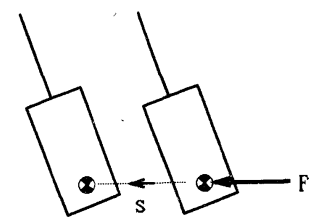

S $X F=0$

conpliance center

Fig. 7 Location of compliance center

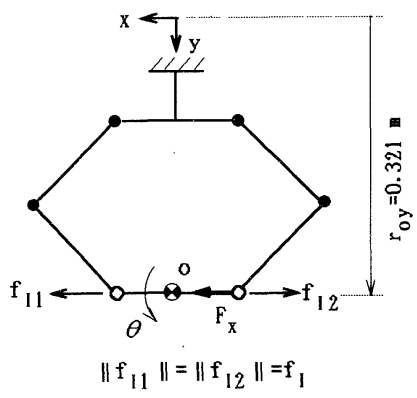

(a) ance center coincide. Notice that, the location of the inherent compliance center depends on the joint stiffnesses, link lengths and the configuration of the mechanism. That is, it can be located at the desired place by changing the link lengths, joint stiffnesses as well as the configuration of the mechanism. For the closed chain mechanism shown in Fig. 1, the link lengths and joint stiffnesses are selected such that, for the configuration of Fig. 4 ( $\mathrm{c}$ ) the inherent compliance center (" $O$ ") is located on the center of the floating link for all the configurations along $y$ axis while $r_{o x}=0$ and $\theta=0$. At these configurations, the additional stiffnesses at point $o$ due to the internal forces have the forms,

$$
\Delta \boldsymbol{k}_{o}^{*}=\left[\begin{array}{ccc}
0 & 0 & \Delta k_{x \theta}^{*} \\
0 & 0 & 0 \\
\Delta k_{\theta x}^{*} & 0 & 0
\end{array}\right], \Delta \boldsymbol{k}_{o}^{* *}=\left[\begin{array}{ccc}
0 & 0 & 0 \\
0 & 0 & 0 \\
0 & 0 & \Delta k_{\theta}^{* *}
\end{array}\right]
$$

This indicates that, the compliance center is no more at the point $o$ because of the non-diagonal elements in $\Delta \boldsymbol{k}_{o}^{*}$. Instead, the compliance center shifts to another point say " $a$ " where

$$
\boldsymbol{K}_{a}=\sum_{i=1}^{2}\left(\boldsymbol{J}_{i}^{T} \boldsymbol{k}_{q i} \boldsymbol{J}_{i}\right)_{a}+\Delta \boldsymbol{k}_{a}^{*}+\Delta \boldsymbol{k}_{a}^{* *}
$$

is diagonal. In other words, by using the internal forces the compliance center of a closed chain mechanism can be shifted from one point to another without changing its inherent stiffness. Figs. 8(b) and 9 (b) show the rotational deflection of the end effector around the point where the external force is applied, for different modes of internal forces. In the case of Fig. 8 ( b), the external force is applied at the inherent compliance center (see Fig. 8 (a)). Therefore, the deflection $\delta \theta=0$ if there is no internal force. However, in the case of Fig. 9 ( $b$ ) the external force

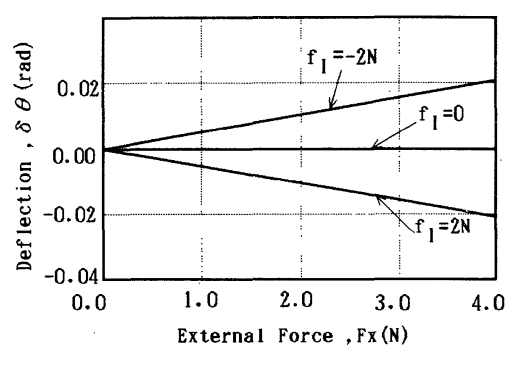

(b)

Fig. 8 Rotational deflection due to the external forces acting at the inherent compliance center 


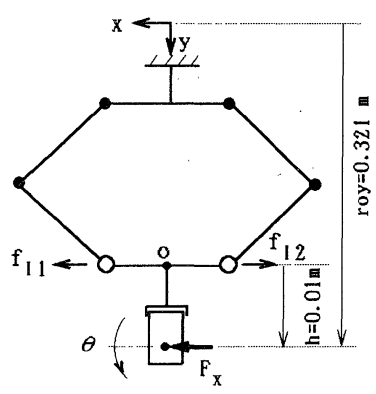

$\left\|f_{11}\right\|=\left\|f_{12}\right\|=f_{1}$

(a)

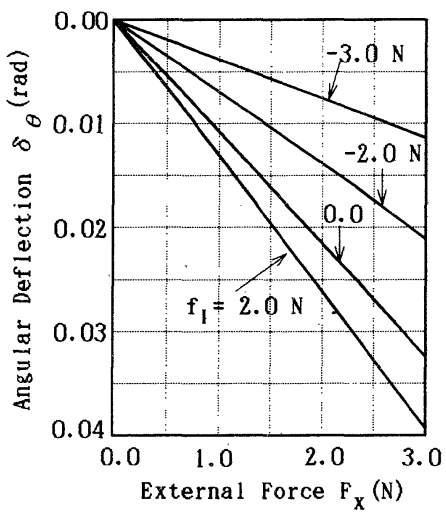

(b)

Fig. 9 Rotational deflection due to the external forces acting away from the inherent compliance center

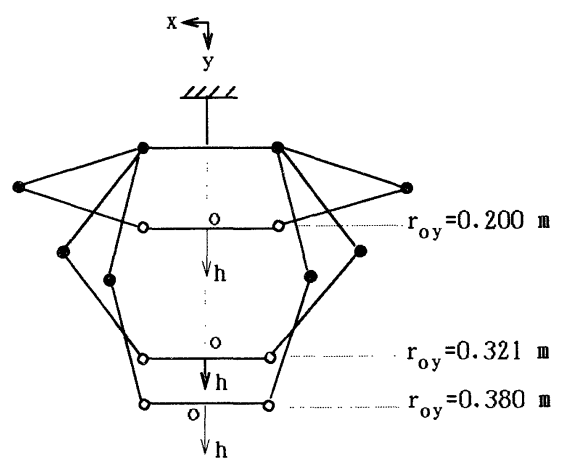

(a)

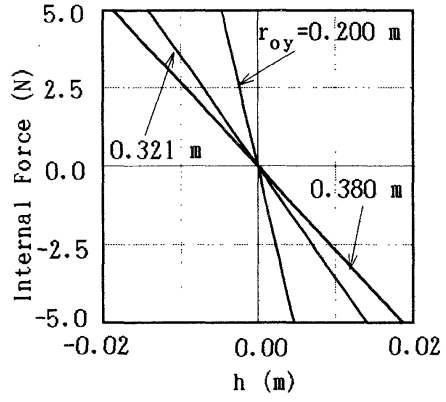

(b)

Fig. 10 Required internal force to realize the desired compliance center

is applied at a point away from the inherent compliance center (see Fig. 9(a)), hence, even for the case of no internal force, there is a rotational deflection indicating that there exists a non-diagonal element in the inherent compliance matrix relating the rotational motion to the lateral force $F_{x}$. As the compressive internal force is increased, the deflection $\delta \theta$ decreases, meaning that the compliance center is approaching to the point where the external force is applied. The results are for the mechanism with the configuration shown in Fig. 4(c). The joint stiffnesses and the link lengths are set to the same values as in section 3 .

The required internal force to shift the compliance center from the inherent compliance center to a new desired point is found by substituting eqs. ( 9 ) and (11) into eq. (20) and solving for the internal force $f_{I}$ that leads to the diagonalization of matrix $\boldsymbol{K}_{a}$,

$$
\begin{gathered}
f_{I}=\frac{4 l \sum_{i=1}^{2} k_{s i x} \sin ^{2}\left(q_{i 1}-q_{i 2}\right)}{l_{o 2} \sum_{i=1}^{2}\left[\sin \left(2 q_{i 2}\right) \cos q_{i 1}+\sin \left(2 q_{i 1}\right) \cos q_{i 2}\right]} h, \\
i=1,2
\end{gathered}
$$

Here, " $h=\left(r_{e y}-r_{o y}\right)$ " is the distance from the inherent compliance center to the desired compliance center. As it is clear from eq. (21), the amount of internal force to obtain the same $h$ will differ for each configuration of the mechanism as well as the link lengths and joint stiffnesses. In other words eq. (21) indicates that, it is possible to change the range of " $h$ " by either changing the joint stiffnesses, the link parameters or the configuration of the mechanism. Also, it is clear that, the amount of internal force to shift the compliance center is strongly related to the length of $l_{o 2}$. As $l_{o 2}$ becomes smaller we need more internal 


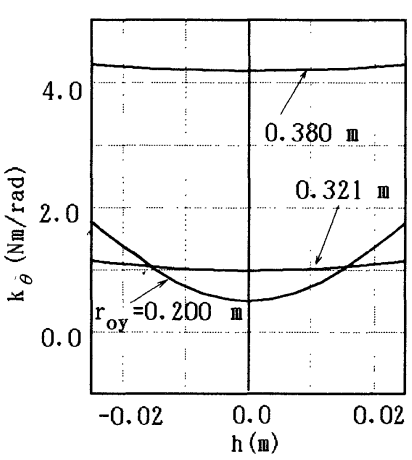

(a) inherent rotational stiffness

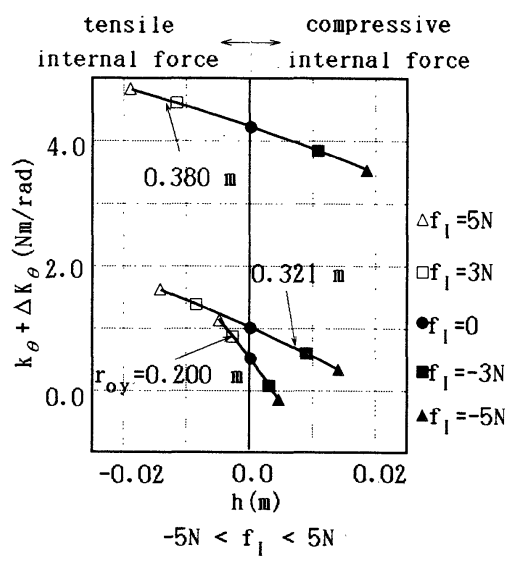

(b) resultant rotational stiffness

Fig. 11 Rotational stiffness at the new compliance centers

force. Equation (21) is plotted in Fig. 10 ( b ) for three different configurations of the mechanism shown in Fig. 10 (a) while the joint stiffnesses and the link lengths are kept constant. As it is clearly seen in Fig. 10 (b), tensile internal forces are required to move the compliance center inward (i.e., $h<0$ ). However, as we have already explained in section 3 , if the tensile internal forces are used, the rotational stiffness will increase, in other words the rotational compliance will decrease, which is not a desirable case. For this reason moving the compliance center inward is not suggested. On the contrary, to move the compliance center outward requires compressive internal forces. This gives us the opportunity to simultaneously increase the rotational compliance at the new compliance center. This can be observed from Figs. 11 (a) and 11 (b) that show the inherent rotational stiffness and the resultant rotational stiffness at the new compliance centers for the same three configurations of Fig. 10 (a). Notice from Fig. 11 ( b ) that, for each configuration, the effect of internal force on shifting the compliance center is different. At the contracted configurations (i.e., when $r_{o y}$ is short), the required internal force to shift the compliance center is bigger as compared to the extended configurations (i.e., when $r_{o y}$ is long). Also, contracted configurations result in low rotational stiffness. Hence, at such configurations, applying large internal forces may violate eq. (16), that is, the total rotational stiffness may become negative (as for the case of $r_{o y}=0.200 m$ in Fig. 11 (b)) and as a result the mechanism will be unstable. The suitable configuration must be decided taking all these factors into account.

\section{Conclusion}

Based on the fact that, the internal forces in closed chain mechanisms generate an additional stiffness which is clearly distinguished from the inherent stiffness of the mechanisms, we showed how to utilize the internal forces in compliance control and shifting the compliance center from one location to another in a planar closed chain mechanism while keeping the inherent stiffness of the mechanism unchanged. However, to achieve the compliance control in all directions and/or to be able to locate the compliance center at any arbitrary location is not possible unless abundant number of redundant actuators are used. To avoid this, we have limited our scope to the cases of the compliance control in rotational directions only. For this reason, we have selected special geometrical configurations of the mechanism that will allow us to control the rotational compliance via internal forces independently from the compliance of the other directions. One such configuration is that of Fig. 4 (c). In this configuration, the links on the arms have the same lengths and the active joints are set to the same stiffness. To operate with such a configuration allows us to use only one redundant actuator for generating the rotational stiffness. This configuration also allows 
us to locate the inherent compliance center at the middle of the floating link. The range of selective compliance center with this configuration is not very large and is limited along the vertical direction ( $y$ axis). Nevertheless, to obtain different ranges is a design problem which will be considered in our future studies. To obtain different ranges for " $h$ " can be achieved by changing the mechanical structure of the mechanism in the form of the link lengths, joint stiffnesses, location of the end effector, etc.

The planar closed chain mechanism that we analyzed in this paper can be used in two ways: either as a parallel manipulator or as a compliance device attached to the end of a serial link manipulator. The concept of this study is more applicable to the latter case, since in this case the deviations in the position and orientation of the mechanism will be very small. Once the desired task is specified, the mechanism can be set to a suitable configuration such that, the small movements will be confined around this configuration. Then, during the task, the rotational compliance of the end effector and/or the compliance center can be changed to the desired value by only controlling the internal forces.

\section{References}

1) J.K. Salisbury: Active Stiffness Control of a Manipulator in Cartesian Coordinates, Proceedings of the IEEE Decision and Control Conference, Albuquerque, NM (1980)

2) K. Yokoi, M. Kaneko and K. Tanie: A Compliance Control Method Suggested by Muscle Network in Human Arms, Proceedings of IEEE International Workshop on Intelligent Robots and Systems, Tokyo, Japan, Oct.31-Nov. 2 (1988)

3) H. Hanafusa and M. A. Adli : Effect of Internal Forces on Stiffness of Closed Mechanisms, Proceedings of '91 ICAR, Fifth International Conference on Advanced Robotics, Pisa, Italy (1991)

4) M. A. Adli, K. Nagai, K. Miyata and H. Hanafusa : Analysis of Internal Force Effect in Parallel Manipula. tors, Transaction of the SICE, 27-11, 1266/1273 (1991)

5) B. J. Yi and R. Freeman: Simultaneous Stiffness Generation and Load Distribution in Redundantly Actuat. ed Mechanisms, Proceedings of ' 91 ICAR, Fifth International Conference on Advanced Robotics, Pisa, Italy (1991)

6) B. J. Yi, R. Freeman and D. Tesar: Open-Loop Stiffness Control of Overconstrained Mechanisms/Robotic
Linkage Systems, Proceedings of IEEE International Conference on Robotics and Automation, Scottsdale, AZ-3, 1340/1345 (1989)

7) B.S. Baker, S. Fortune and E. Grosse: Stable Prehension with a Multi-fingered Hand, Proceedings of IEEE International Conference on Robotics and Automation, St. Louis, 570/575 (1985)

8) V. Nguyen: The Synthesis of Stable Grasps in the Plane, Proceedings of IEEE International Conference on Robotics and Automation, San Francisco, 884/889 (1986)

9) M. R. Cutkosky and I. Kao: Computing and Controlling the Compliance of a Robotic Hand, IEEE Transactions on Robotics and Automation, RA-5-2, 151/165 (1989)

10) M. Kaneko, N. Imamura, K. Yokoi, and K. Tanie: A Realization of Stable Grasp Based on Virtual Stiffness Model by Robot Fingers, Proceedings of IEEE International Workshop on Advanced Motion Control, Yokohama, 156/163 (1990)

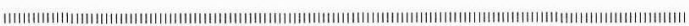
Mehmet Arif ADLI

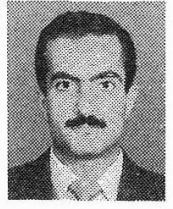

M. Arif Adli received the B. S. degree from Middle East Technical University, Ankara, Turkey in 1986, and M.S. degree from Ritsumeikan University, Kyoto, Japan in 1989, both in mechanical engineering. $\mathrm{He}$ is currently working toward the $\mathrm{Ph}$. D. degree at the same university. His research concerns analysis and control of closed chain mechanisms. Mr. Adli is a member of Robotics Society of Japan.

\section{Hideo Hanafusa (Member)}

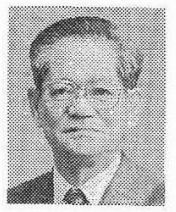

Hideo Hanafusa received B.E from Department of Aeronautical Engineering, Kyoto University, in 1945. After teaching at Osaka Municipal University and Kyoto Institute of Polytechnics, he moved to Kyoto University and worked as a professor at the Faculty of Engineering from 1966 to 1986 . Since 1986 he has been with Ritsumeikan University where he is a professor at the Faculty of Science and Engineering. His research activities include hydraulic servo systems, and, mechanisms and control of robots. Professor Hanafusa is a member of Robotics Society of Japan.

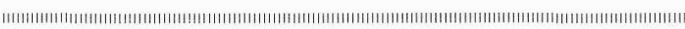

